\title{
The effect of lateral carrier diffusion on the modulation response of a semiconductor laser
}

\author{
D. Wilt, K. Y. Lau, and A. Yariv \\ California Institute of Technology, Pasadena, California 91125
}

(Received 30 March 1981; accepted for publication 30 April 1980)

\begin{abstract}
The effect of lateral carrier diffusion upon the modulation characteristics of the semiconductor laser is investigated. A self-consistent analysis of the spatially dependent rate equations is performed using a finite element model. The transverse junction stripe laser is treated as an example and a comparison is made between lateral carrier diffusion and spontaneous emission as damping mechanisms for the resonance peak. Experimental results bear out the conclusion that the relaxation resonance in this device is damped mainly by lateral carrier diffusion. In addition, a simple analytic result is presented which illustrates qualitatively the effect of lateral carrier diffusion upon such devices. The conclusion from this result is that lateral carrier diffusion serves to damp the relaxation resonance in the semiconductor laser quite well, but probably will not serve to improve the upper limit on modulation frequency as might have been suspected.
\end{abstract}

PACS numbers: 42.55.Px, 42.60.Fc

Semiconductor lasers are potentially devices of great use for optical communications systems based upon their small size, high efficiency, and high speed for direct modulation. Thus, methods for improving the modulation response of these devices such as damping of the relaxation resonance are topics of active current interest.

Several authors have investigated the effect of lateral carrier diffusion upon the modulation response of the semiconductor laser..$^{1-5}$ These investigations have centered mainly upon the time domain response of the laser to a step change in input current, ${ }^{2.3}$ or the small signal frequency response, ${ }^{1,4.5}$ and have indicated that the transverse diffusion of carriers can improve considerably the modulation reponse of the laser. This improvement comes mainly in the damping of the relaxation oscillations of the laser which usually occurs within a time scale of nanoseconds after the current step, or in the damping of the resonance peak in the small signal frequency response at approximately $1-2 \mathrm{GHz}$.

The purpose of this paper is to present a simple, selfconsistent model of the effect of lateral carrier diffusion upon the semiconductor laser to illustrate both its theoretical and practical limits in the improvement of the modulation response of these devices. This model is formulated in the frequency regime so that the analog small signal response of the device is made available. The general model developed is applied to the case of the TJS (transverse junction stripe) laser ${ }^{b}$ as an example, and the effect of both the carrier diffusion and the spontaneous emission factor in the damping of the relaxation resonance of this device are compared with experiment. The TJS laser is chosen as an example since its injection can be modelled by a $\delta$ - function spatial dependence, eliminating the question of the lateral distribution of injected current, and clarifying the contribution of lateral carrier diffusion to the device.

The starting point of this analysis is the spatially-dependent rate equations, using the assumption that only a single carrier need be considered (for example, holes). We also assume that the active layer in the laser is thin, so that all the physical variables are averaged over this dimension. The la- ser is assumed to oscillate in a single lateral mode.

$$
\begin{aligned}
\frac{\partial n}{\partial t}= & \frac{n}{\tau_{s}}+D \frac{\partial^{2} n}{\partial x^{2}}-\frac{S}{\hbar \omega} \frac{\Gamma}{d} F a\left(n-n_{i}\right)+\frac{j}{e d} \\
\frac{d S}{d t}= & v S \Gamma \int_{-\infty}^{\infty} F a\left(n-n_{t}\right) d x-\frac{S}{\tau_{p}} \\
& +\beta v \hbar \omega d \int_{\infty}^{\infty} \frac{n}{\tau_{s}} d x .
\end{aligned}
$$

In these equations, $n$ is the inversion density, $\tau_{s}$ is the spontaneous lifetime, $D$ is the lateral diffusion constant, $S$ is the power in the single lasing mode, $\hbar \omega$ is the photon energy, $a$ is the gain coefficient, and $n$, is the inversion required for transparency, so that the optical gain is a $\left(n-n_{t}\right), F$ is the lateral intensity profile of the optical mode, assumed normalized, $\Gamma$ is the intensity confinement factor normal to the active region, $j$ is the injected current density, $e$ is the electronic charge, $d$ is the active layer thickness, $v$ is the group velocity of the optical mode, $\tau_{p}$ is the photon lifetime, and $\beta$ is a coupling coefficient for spontaneous emission into the optical mode.

The spontaneous emission factor is treated in a very simple manner here. To treat spontaneous emission into the lasing mode properly would involve a much more complex treatment which is unnecessary for this calculation. This is because the major contribution of spontaneous emission to the modulation characteristics comes from the background it adds to the optical power. Spontaneous emission could also be considered by adding a constant term $S_{\text {spont }} / \tau_{p}$ to Eq. (2).

These equations are normalized in the following manner. Time is normalized to $\tau_{5}$, distance is normalized to the diffusion length, $\left(D \tau_{s}\right)^{1 / 2}, n$ is normalized to $n_{t}, S$ is normalized to $\hbar \omega d\left(D \tau_{s}\right)^{1 / 2} / a \Gamma \tau_{s}$, and $j$ is normalized to $e d n_{t} / \tau_{s}$. With this choice of normalization, the rate equations appear as

$$
\frac{\partial n}{\partial t}=-n+\frac{\partial^{2} n}{\partial x^{2}}-S F(n-1)+j
$$


$\frac{d S}{d t}=a_{1} a_{2} S \int_{-\infty}^{\infty} F(n-1) d x-a_{1} S+\beta a_{1} a_{2} \int_{-\infty}^{\infty} n d x$

The two parameters $a_{1}$ and $a_{2}$ are

$$
\begin{aligned}
& a_{1}=\frac{\tau_{s}}{\tau_{p}}, \\
& a_{2}=\Gamma_{a n} v \tau_{p} .
\end{aligned}
$$

With reasonable values of the various parameters, $\tau_{s}=3 \mathrm{nS}$, $D=10 \mathrm{~cm}^{2} / \mathrm{s}, \hbar \omega=1.43 \mathrm{eV}, \Gamma=0.5, d=0.2 \mu \mathrm{m}$,

$a=10^{-18} \mathrm{~cm}^{2}, n_{t}=2 \cdot 10^{18} \mathrm{~cm}^{-3}, v=10^{10} \mathrm{~cm} / \mathrm{s}, \tau_{p}=2 \mathrm{pS}$. The values for these parameters are approximately $1.5 \cdot 10^{3}$ for $a_{1}$ and 2 for $a_{2}$. In addition we have the condition

$$
v \tau_{p}=L /[\alpha L+\ln (1 / R)],
$$

where $L$ is the diode length, $\alpha$ is a distributed loss, and $R$ is the mirror reflectivity of the device.

In a qualitative sense, one might argue that lateral carrier diffusion in a device with a narrow optical mode and a very restricted current injection would increase the maximum modulation speed of the laser. This argument claims that the effective carrier lifetime in the device is shortened by the fact that carriers that diffuse away from the optical-mode region are lost to stimulated emission. This, and the fact that the maximum modulation rate depends upon carrier lifetime, would seem to indicate that such a diffusion dominated laser would be a faster device. In addition, one might expect that lateral carrier diffusion serves to damp the relaxation oscillations in the laser diode, since the exchange of energy between the photons and inverted carriers responsible for these oscillations is damped strongly by the gain and loss of carriers that diffuse into and out of the optical-mode region.

An analytic result can be derived from the rate equations that indicates to what extent these qualitative arguments are true. One simple limiting case is that where the optical mode and current injection have no spatial dependence. Another limiting case, the one of most interest here, is that where the current injection and optical mode are both $\delta$ functions in the lateral direction. In either case, we will derive small signal modulation transfer functions, and a comparison reveals the extent to which the above assertions are true. To simplify the analytic results and to clarify the contribution of diffusion, we will assume the spontaneous emis' sion coupling $\beta$ to be zero.

The steady-state solution to the spatially uniform case is then

$$
\begin{aligned}
& \left(n_{0}-1\right)=1 / a_{2}, \\
& S_{0} F=a_{2}\left(j_{0}-j_{\mathrm{th}}\right), \\
& j_{\mathrm{th}}=1+\left(1 / a_{2}\right),
\end{aligned}
$$

where zero subscripts indicate steady-state values. Note that a power density, $S_{0} F$, is given, where $F$ is a constant, and the threshold current is defined as $j_{\mathrm{th}}$. The small signal equations for the spatially uniform case are, assuming $n=n_{0}+n_{1} e^{i \omega t}$, $S=S_{0}=S_{1} e^{i \omega t}$, and $j=j_{0}+j_{1} e^{i \omega t}$,

$$
\begin{aligned}
& i \omega n_{1}=-n_{1}-S_{10} F n_{1}-S_{1} F\left(n_{0}-1\right)+j_{1}, \\
& i \omega S_{1}=a_{1} a_{2} S_{0} n_{1}+a_{1} a_{2} S_{1}\left(n_{0}-1\right)-a_{1} S_{1} .
\end{aligned}
$$

Using the steady-state relationships, (8)-(10), these simplify to

$$
\begin{aligned}
& n_{1}\left(i \omega+1+S_{0} F\right)+S_{1} F\left(1 / a_{2}\right)=j_{1}, \\
& i \omega S_{1} F=a_{1} a_{2} S_{0} F n_{1} .
\end{aligned}
$$

Solving these relationships yields

$$
S_{1} F=a_{2} j_{1} \frac{1}{1+i \omega / a_{1}\left\{1+\left[(1+i \omega) / S_{0} F\right]\right\}} .
$$

This transfer function represents a two-pole lowpass response, with a characteristic frequency of

$$
\omega_{r}=\left(a_{1} S_{0} F\right)^{1 / 2} .
$$

With reasonable values of $a_{1}, a_{2}$, and the power density $S_{0} F$, this resonance lies in the microwave range of $1-2 \mathrm{GHz}$. However, the resonance also has a typical amplitude of 10 $\mathrm{dB}$ over the de value. This represents a considerable problem to the use of this device in a high-speed communications system. This response is plotted as a function of frequency in Fig. 1 for the values of the parameters as specified above. $S_{0} F$ takes the values of $0.1,0.3,1$, and $3 \mathrm{~mW} / \mu \mathrm{m}$ in these curves.

In the case where the current injection and the optical mode have $\delta$ function profiles,

$$
\begin{aligned}
& j(x)=A \delta(x), \\
& F(x)=\delta\left(x-x_{0}\right),
\end{aligned}
$$

the steady-state solution to the equations above, neglecting spontaneous emission, is

$$
\begin{aligned}
& n_{0}\left(x_{0}\right)=1+\frac{1}{a_{2}}, \\
& n_{0}(x)=\frac{A_{0}}{2} e^{-|x|}-\frac{S_{0}}{2 a_{2}} e^{-\mid x-x_{01},}, \\
& S_{0}=a_{2} e^{-\left|x_{10}\right|}\left(A_{0}-A_{\mathrm{th}}\right), \\
& A_{\mathrm{th}}=2 e^{\mid x_{11}}\left(1+\frac{1}{a_{2}}\right)
\end{aligned}
$$

where the lasing threshold $A_{\mathrm{th}}$ has been defined. Note that this threshold is just $e^{\mid x_{i, i}}$ times the threshold for a two-diffu-

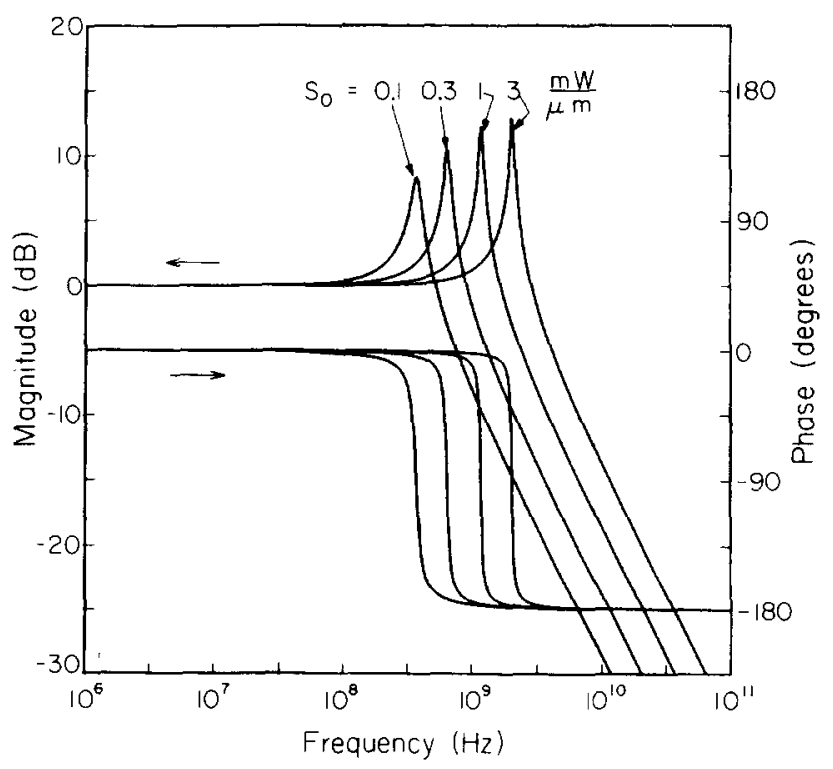

FIG. 1. Small signal transfer function for the spatially uniform laser. 


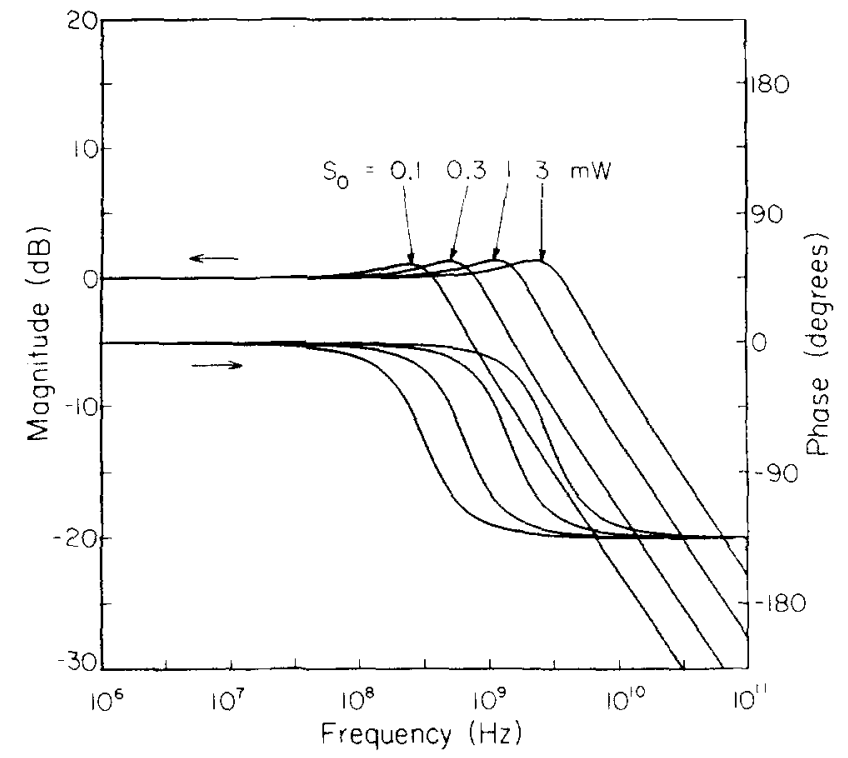

FIG. 2. Small signal transfer function for the $\delta$-function laser.

sion length wide uniform laser. The small signal equations for this case are then

$$
\begin{aligned}
i \omega n_{1}= & -n_{1}+\frac{d^{2} n_{1}}{d x^{2}}-S_{1}\left(n_{0}-1\right) \delta\left(x-x_{0}\right) \\
& -S_{1} n_{1} \delta\left(x-x_{0}\right)+A_{1} \delta(x), \\
i \omega S_{1}= & a_{1} a_{2} S_{1}\left[n_{01}\left(x_{0}\right)-1\right]+a_{1} a_{2} S_{0} n_{1}\left(x_{0}\right)-a_{1} S_{1} .
\end{aligned}
$$

Again, using the steady-state relationships, (19)-(22), we can reduce these relationships to

$$
i \omega S_{1}=a_{1} a_{2} S_{0} n_{1}\left(x_{0}\right)
$$

$$
\begin{aligned}
& \frac{d^{2} n_{1}}{d x^{2}}-n_{1}(1+i \omega) \\
& \quad=A_{1} \delta(x)-S_{0}\left(1+\frac{a_{1}}{i \omega}\right) n_{1}\left(x_{0}\right) \delta\left(x-x_{0}\right),
\end{aligned}
$$

A solution to these equations which must be made self-consistent is

$$
\begin{aligned}
& n_{1}(x)=\frac{A_{1}}{2(1+i \omega)^{1 / 2}} e^{-|x|(1+i(-))^{\prime} \cdot,}, \\
& -\frac{S_{0}\left(1+a_{1} / i \omega\right)}{2(1+i \omega)^{1 / 2}} n_{1}\left(x_{0}\right) e^{-x \cdot x_{0}|1+i(\omega)|^{\prime} .} .
\end{aligned}
$$

The requirement of self-consistency yields

$$
n_{1}\left(x_{0}\right)=\frac{A_{1}}{2} e^{-\left|x_{i}\right|(1+i \omega\}^{1 / 2}} \frac{1}{(1+i \omega)^{1 / 2}+\left(S_{0} / 2\right)\left(1+a_{1} / i \omega\right)}
$$

With some simplification and the substitution of Eq. (25) this gives

$$
S_{1}=a_{2} A_{1} \frac{e^{\left|x_{\mathrm{u}}\right| 1+i+\left.i(\omega)\right|^{\prime \prime}}}{\left(1+i \omega / a_{1}\right)\left\{1+\left[(1+i \omega)^{1 / 2} /\left(S_{0} / 2\right)\right]\right\}} .
$$

The resemblance between Eqs. (29) and (15) is remarkable. The behavior of this transfer function is plotted in Fig. 2, again for the parameters chosen earlier, and with the offset $x_{0}$ chosen to be zero. The parameter $S_{0}$ takes on the values $0.1,0.3,1$, and $3 \mathrm{~mW}$. This transfer function has slightly improved frequency response over the uniform case, manifested mostly in the reduced resonance peaks. This is achieved, however, at the price of slightly worse phase response in the region near the resonance.

This transfer function represents the maximum contribution of diffusion to the modulation behavior of the semiconductor laser. Any other case should lie in the intermediate region between the spatially uniform case and this $\delta$ function laser. It is most interesting to note that this limiting case has an analytic solution which does not show pathological behavior such as an infinite frequency response. In fact, even with the infinite power density represented by the $\delta$ function optical mode, this laser has an upper limit on modulation frequency which is quite similar to the spatially uniform case. Thus it appears that while lateral carrier diffusion may be expected to improve the damping of the relaxation resonance, it cannot be expected to improve greatly the upper modulation frequency limit of the semiconductor laser.

This exact analytic solution to the rate equations may be compared to the approximate calculations presented by Furuya et al. ${ }^{4}$ and Thompson ${ }^{5}$ in the limit of narrow stripe widths. In Ref. (4), the zero stripe width case shows a modulation resonance peak height of approximately $12 \mathrm{~dB}$ over the low-frequency value. In Ref. (5), using the laterally unconfined results appropriate to the situation analyzed here, the small stripe width limit shows a suppressed modulation resonance peak similar to the exact result, but also a modulation response at high frequencies approximately $5 \mathrm{~dB}$ below the low-frequency value. Neither of these approximate results is in agreement with the exact result, Eq. (29).

To analyze the case where the injected current and the optical mode are allowed to assume arbitrary profiles, numerical analysis of the rate Eqs. (3) and (4) is required. First, the steady-state solution of these equations is found, and using this solution, small signal equations for the system are derived. Since the steady-state equations are nonlinear, their solution can be quite tedious. However, if we assume the optical-mode profile $F$ to be fixed and the profile of the injected current $j$ to be fixed so that $j=A G(x)$, where $G$ is a fixed function and $A$ is a scalar, this calculation can be reduced to the solution of a linear system.

With the output power $S_{1)}$ assumed, the equations to be solved for the steady-state solution are

$$
\begin{aligned}
& -n+\frac{\partial^{2} n}{\partial x^{2}}-S_{4} F(n-1)+A G=0 \\
& a_{1} a_{2} S_{0} \int_{\infty}^{\infty} F(n-1) d x-a_{1} S_{0}+\beta a_{1} a_{2} \int^{\infty} n d x=0
\end{aligned}
$$

These equations are both linear in the unknowns, $n$ and $A$. With the assumption of a finite element variational form for $n$ involving a one-dimensional grid where the values of $n$ at the nodes are the variational parameters and using linear interpolation between nodes, these two equations are transformed into a linear algebraic system which may be solved quite simply to yield both the nodal values of $n$ and the scalar current $A$.

In the small signal analysis, the equations are already linear in all the unknowns, and we simply assume a value for 


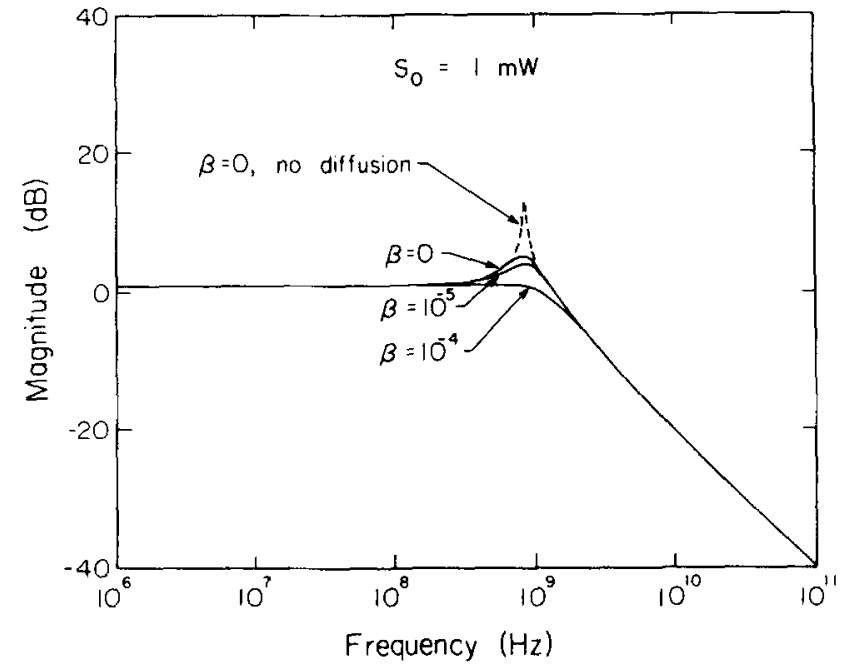

FIG. 3. Magnitude of small signal transfer function for TJS laser, showing dependence upon the spontaneous emission coupling factor.

the small signal output power $S_{1}$, assume a finite element form for the small signal carrier distribution $n_{1}$, and solve the resulting linear algebraic system for the nodal values of $n_{1}$ and the small signal scalar current $A_{1}$.

The general model developed above may be applied to any double heterostructure semiconductor laser where the optical-mode profile, $F(x)$, and the injected current profile $G(x)$ can be assumed known. The calculation presented in this paper has been performed for the TJS laser, and the results are shown in Figs. 3 through 6. The TJS laser was taken as the test device because of its simple electrical and optical structure and small lateral dimensions. Since it is a lateral homojunction device, the current injection profile $G$ can be taken to have a $\delta$ function form. This eliminates the problem of determining how the current distribution varies with steady-state optical power output or modulation frequency. In addition, this device has a built-in index profile in the lateral dimension that defines the lateral optical-mode

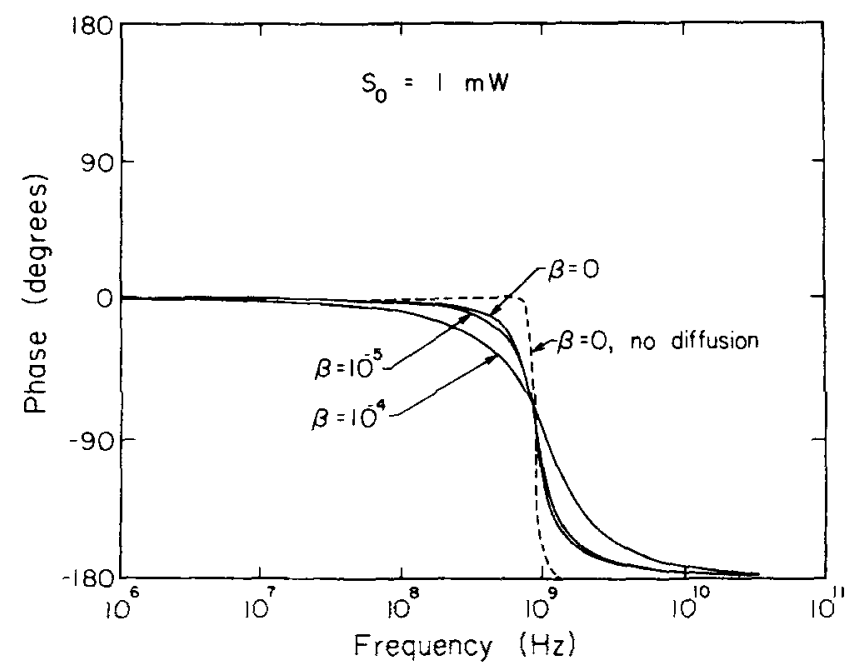

FIG. 4. Phase of small signal transfer function for TJS laser, showing dependence upon the spontaneous emission coupling factor.

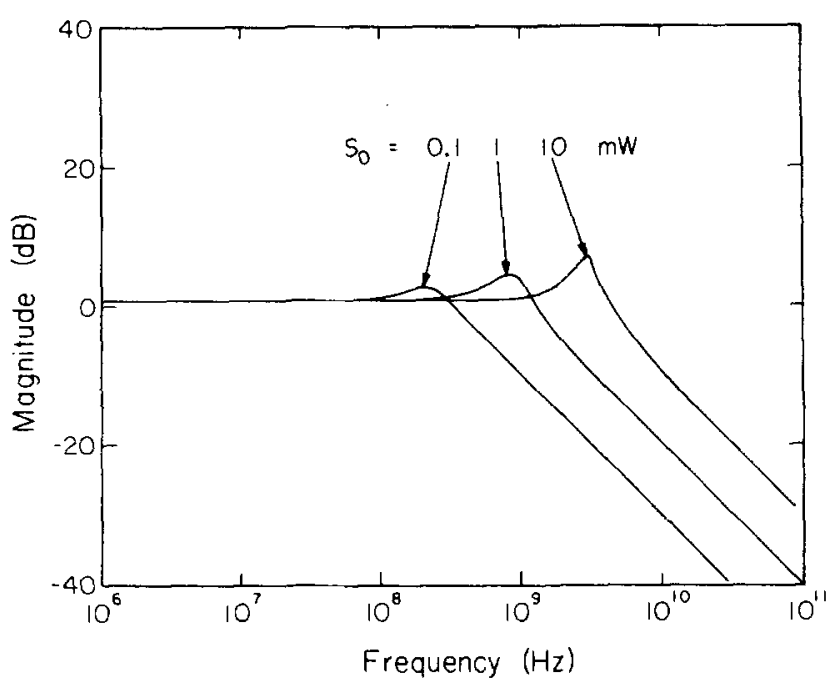

FIG. 5. Magnitude of small signal transfer function for TJS laser, showing dependence upon the steady-state output power.

profile $F$. The small lateral dimensions of this device (typically a few $\mu \mathrm{m}$ ) make lateral carrier diffusion an important contribution to its dynamic behavior. In the calculation, the parameters for the device were taken to be $\alpha=50 \mathrm{~cm}^{-1}$, $R=0.3, \Gamma=0.6, \hbar \omega=1.43 \mathrm{eV}, a=10^{-18} \mathrm{~cm}^{2}, d=0.2$ $\mu \mathrm{m}, L=250 \mu \mathrm{m}, n_{t}=2 \cdot 10^{18} \mathrm{~cm}^{-3},\left(D \tau_{s}\right)^{1 / 2}=3 \mu \mathrm{m}$, $\tau_{s}=3 \mathrm{nS}$, and $v=0.83 \cdot 10^{10} \mathrm{~cm} / \mathrm{s}$. This gives for the parameters $a_{1}=2500$ and $a_{2}=1.2$. The lateral mode intensity profile assumed for this device is

$$
F(x)=\frac{1}{(2 \pi)^{1 / 2} w} e^{-\left[\mid x-x_{n}+1 / 2 w^{*}\right]},
$$

where $x_{\text {of }}$ is the offset between the center of the optical mode and the diode junction, and $w$ is the width of the lasing mode, both assumed to be $0.6 \mu \mathrm{m}$. The origin is taken to lie at the diode junction. The small signal modulation transfer functions calculated for these parameters, a power output from the device of $1 \mathrm{~mW}$, and various values of the spontaneous

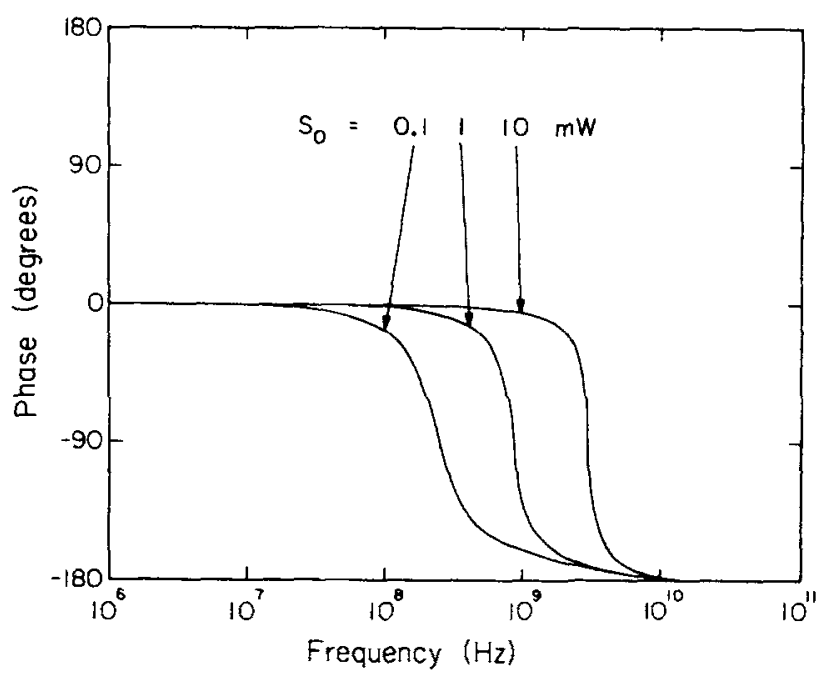

FIG. 6. Phase of small signal transfer function for TJS laser, showing dependence upon the steady-state output power. 

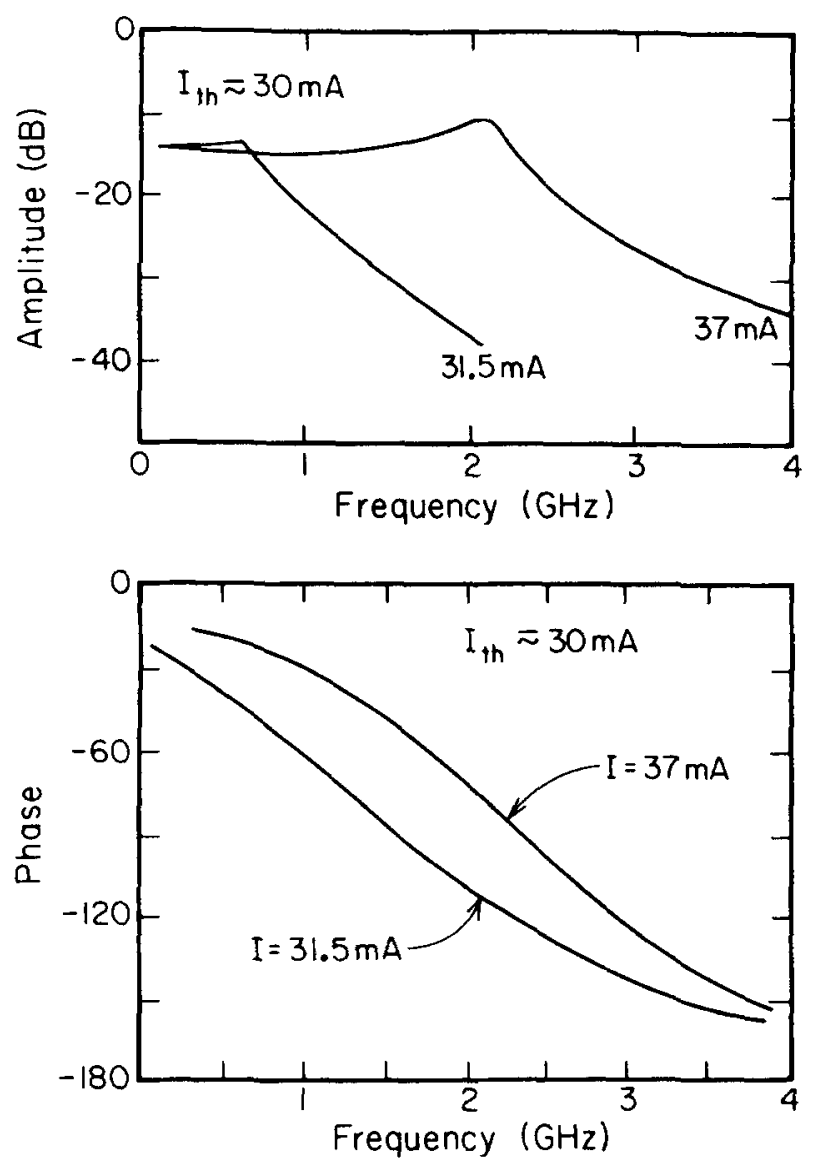

FIG. 7. Measured amplitude and phase response of a TJS laser.

emission coupling factor are shown in Figs. 3 and 4. The interesting feature of these curves is the supression of the relaxation oscillation resonance caused by the lateral diffusion of carriers in the device. Compared with the spatially uniform case, the height of the resonance is lowered by approximately $9 \mathrm{~dB}$ by the action of lateral diffusion. The additional contribution of spontaneous emission into the lasing mode can be seen to be appreciable only for relatively large spontaneous emission coupling factors of $10^{-4}$. In Figs. 5 and 6, the behavior of the transfer function as a function of optical output power is calculated for a spontaneous emission coupling of zero. The optical output powers are $0.1 \mathrm{~mW}$ for the lowest curve, $1 \mathrm{~mW}$ for the center curve, and $10 \mathrm{~mW}$ for the upper curve.

Figure 7 shows the measured small signal transfer function for a TJS laser. It should be noted that the vertical scale in this measurement is twice as large as in the theoretical calculations. This is due to the fact that the measurement was done with a square law detector, a silicon avalanche photodiode (APD). The resonance peak in the amplitude response of this device has a magnitude of approximately $5 \mathrm{~dB}$ on this scale, or $2.5 \mathrm{~dB}$ on the scale of the theoretical curves. This corresponds well with the calculations if a spontaneous emission factor of approximately $3 \cdot 10^{-5}$ is assumed. This is a reasonable value to assume for spontaneous emission coupling in this type of device. It is quite difficult to draw any more information from this experimental measurement as it contains also the frequency response of the measurement system, including the APD response which has a 3-dB cutoff frequency of approximately $1.8 \mathrm{GHz}$.

The measured phase response of the system also supports the diffusion damped model of the laser, since it displays a gradual rolloff, rather than the sharp transition of the spatially uniform laser.

The model of the TJS laser, then, as a diffusion damped device can be seen to be verified quite well. This device should be quite useful in both analog and digital information transmission systems, where its well-damped resonance would cause minimal interference with modulated information.

This calculation and experimental result may be compared with the calculations presented by Furuya $\mathrm{et} \mathrm{al}^{4}{ }^{4}$ and Thompson. ${ }^{5}$ In Ref. (4), a modulation resonance peak of approximately $10 \mathrm{~dB}$ is predicted for this structure. This is larger than the resonance peak calculated or measured in this work. In Ref. (5), using the laterally unconfined approximation, a completely damped resonance peak and broad $-5 \mathrm{~dB}$ response at high frequencies is predicted. That result is not in agreement with this calculation or experiment.

In conclusion, a model of diffusion effects on the small signal modulation characteristics of the semiconductor laser has been presented which shows both the theoretical limits and practical behavior of the diffusion dominated laser. It can be seen that lateral carrier diffusion is a useful mechanism for the control of the relaxation resonance in this device. It has also been shown that the use of lateral carrier diffusion to improve the upper limit on modulation frequency is not practical. In addition, the model of the TJS laser as a diffusion damped laser has been shown to account for the experimental small signal modulation function of this device.

\section{ACKNOWLEDGMENTS}

This work was supported by the Office of Naval Research and the National Science Foundation.

'T. Ikegami, First European Conference on Optical Fibre Communication, IEE London, September, 1975 (unpublished).

${ }^{2} J$. Buus and M. Danielsen, IEEE J. Quantum Electron. QE-13, 669 (1977). ${ }^{3} \mathrm{~N}$. Chinone, K. Aiki, M. Nakamura, and R. Ito, IEEE J. Quantum Electron. QE-14, 625 (1978).

${ }^{4}$ K. Furuya, Y. Suematsu, and T. Hong, Appl. Opt. 17, $1949\{1978\}$

${ }^{5}$ G. H. B. Thompson, Physics of Semiconductor Laser Devices (Wiley, New York, 1980), Sec. 7.3.4.

${ }^{6} \mathrm{H}$. Namizaki, H. Kau, M. Ishii, and A. Ito, J. Appl. Phys. 45, 2785 (1974). 